\title{
SOME GENERALIZED FIBONACCI DIFFERENCE SPACES DEFINED BY A SEQUENCE OF MODULUS FUNCTIONS
}

\author{
Gulsen Kılınc and Murat Candan
}

Abstract. This paper submits the sequence space $l(\widehat{F}(r, s), \mathcal{F}, p, u)$ and $l_{\infty}(\widehat{F}(r, s), \mathcal{F}, p, u)$ of non-absolute type under the domain of the matrix $\widehat{F}(r, s)$ constituted by using Fibonacci sequence and non-zero real number $r, s$ and a sequence of modulus functions. We study some inclusion relations, topological and geometric properties of these spaceses. Further, we give the $\alpha-\beta$ - and $\gamma$-duals of said sequence spaces and characterization of the classes $(l(\widehat{F}(r, s), \mathcal{F}, p, u), X)$ and $\left(l_{\infty}(\widehat{F}(r, s), \mathcal{F}, p, u), X\right)$. Keywords: Sequence space, Fibonacci sequence, Modulus functions.

\section{Introduction}

Construction of new sequence spaces and defining their topological and algebraic properties have a big importance in summability theory. Until now, many sequence spaces were defined by many different ways. One of them is to use the matrix domain of a special triangle which was recently studied by many researchers. The cause of this choice is various characteristics which matrix domains of triangles own. For instance, If $A$ is a triangle matrix and $\lambda$ is a $B K$-space, then $\lambda_{A}$ is a $B K-$ space with the norm given by $\|x\|_{\lambda_{A}}=\|A x\|_{\lambda}$ for all $x \in \lambda_{A}$. By a sequence space, It is understood that a linear subspace of the space $w=\mathbb{C}^{\mathbb{N}}$ of all complex sequences which includes $\phi$, the set of all finitely non-zero sequences, where $N=\{0,1,2, \ldots\}$. We illustrate by $l_{\infty}, c_{0}, c$ and $l_{p}$ for the classical sequence spaces of all bounded, null convergent, convergent and absolutely $p$-summable sequences respectively, where $1 \leq p<\infty$. These spaces are Banach spaces with following norms: $\|x\|_{\ell_{\infty}}=\|x\|_{c}=$ $\|x\|_{c_{0}}=\sup _{k}\left|x_{k}\right|,\|x\|_{b s}=\|x\|_{c s}=\sup _{n}\left|\sum_{k=1}^{n} x_{k}\right|$, and $\|x\|_{\ell_{p}}=\left(\sum_{k}\left|x_{k}\right|^{p}\right)^{\frac{1}{p}}$, while $\phi$ is not Banach space according to any norm. For sake of brevity, here and after the summation without limits runs from 1 to $\infty$. A sequence space $\lambda$ with a linear topolgy is entitled a $K$-space if each of the maps $p_{i}: \lambda \rightarrow \mathbb{C}$ defined by $p_{i}(x)=x_{i}$ is continuous for all $i \in \mathbb{N}$; A $K$-space is entitled an $F K$-space if $\lambda$

Received October 25, 2016; accepted January 15, 2017

2010 Mathematics Subject Classification. 46A45; 46B45, 46B20 
is a complete linear metric space; An $F K$-space whose topology is normable is entitled a $B K$-space [10]. Let $A=\left(a_{n k}\right)$ be a triangle matrix, that is $a_{n k}=0$ for $k>n$ and $a_{n n} \neq 0$ for all $n \in \mathbb{N}$. The equality $A(B x)=(A B) x$ is supplied by the triangle matrices $A, B$ and a sequence $x$. Furthermore, a triangle matrix $A$ has an inverse $A^{-1}$ which is also a triangle matrix and unique such that for each $x \in w, x=A\left(A^{-1} x\right)=A^{-1}(A x)$. Now, let us give description of matrix domain of an infinite matrix due to mentioned significance. The domain $X_{A}$ of an infinite matrix $A$ which is a sequence space is described by

$$
X_{A}=\left\{x=\left(x_{k}\right) \in w: A x \in X\right\},
$$

in a sequence space $X$. The new sequence space $X_{A}$ produced by the infinite matrix $A$ from the space $X$ is the expansion or contraction of the original space $X$. Details can be seen in [3]. Many special limitation method were used for this aim. One of them is Fibonacci matrix.

Let $X, Y$ be any two sequence spaces. Given any infinite matrix $A=\left(a_{n k}\right)$ of real numbers $a_{n k}$, where $n, k \in \mathbb{N}$. For any sequence $x, A$-transform of $x$ is written as $A x=\left((A x)_{n}\right)$. If it is $A$-transform of $x$, it means that $(A x)_{n}=\sum_{k} a_{n k} x_{k}$ converges for each $n \in \mathbb{N}$. If $x \in X$ implies that $A x \in Y$ then $A$ is called a matrix mapping from $X$ into $Y$ and is denoted by $A: X \rightarrow Y$. We illustrate the class of all infinite matrices such that $A: X \rightarrow Y$ by $(X: Y)$.

A linear topological space $X$ over the real field $\mathbb{R}$ is called a paranormed space, if there is subadditive function $g: X \rightarrow \mathbb{R}$ such that $g(\theta)=0, g(x)=g(-x)$, $\left|\alpha_{n}-\alpha\right| \rightarrow 0$ and $g\left(x_{n}-x\right) \rightarrow 0$ imply $g\left(\alpha_{n} x_{n}-\alpha x\right) \rightarrow 0$ for all $\alpha \in \mathbb{R}$ and all $x \in X$, where $\theta$ is the zero vector in the linear space $X$. A paranorm $p$ for which $p(x)=0$ implies $x=0$ is described a total paranormed space. It is well known that the metric of any linear metric space is given by some total paranorm.

Let us suppose that $\left(p_{k}\right)$ be a bounded sequence of certainly positive real numbers with $\sup p_{k}=H$ and $M=\max \{1, H\}$ and $1 / p_{k}+1 / q_{k}^{\prime}=1$ provided $1<$ inf $p_{k} \leq H<\infty$. The linear spaces $\ell_{\infty}(p)$ and $\ell(p)$ were described by Maddox in [21, 22] (see also Simons [27] and Nakano [25]) as follows:

$$
\ell(p)=\left\{x=\left(x_{k}\right) \in w: \sum_{k}\left|x_{k}\right|^{p_{k}}<\infty\right\},
$$

and

$$
\ell_{\infty}(p)=\left\{x=\left(x_{k}\right) \in w: \sup _{k \in \mathbb{N}}\left|x_{k}\right|^{p_{k}}<\infty\right\}
$$

which are the complete spaces paranormed with

$$
h_{1}(x)=\left(\sum_{k}\left|x_{k}\right|^{p_{k}}\right)^{1 / M}
$$


and

$$
h_{2}(x)=\sup _{k \in \mathbb{N}}\left|x_{k}\right|^{p_{k} / M} \text { iff } \inf p_{k}>0
$$

respectively.

The difference sequence spaces had been acquainted by Kizmaz in 1981. He introduced this notion as follow: For $\lambda \in\left\{\ell_{\infty}, c, c_{0}\right\}, \lambda(\Delta)$ consisting of the sequences $x=\left(x_{k}\right)$ such that $\left(x_{k}-x_{k+1}\right) \in \lambda$ is called the difference sequence spaces [17]. From then, many author used this concept greatly to define new sequence space. Some of them are here. The difference spaces $b v_{p}$ consisting of the sequences $x=\left(x_{k}\right)$ such that $\left(x_{k}-x_{k-1}\right) \in \ell_{p}$ have been studied in the case $0<p<1$ by Altay and Başar [2], and in the case $1 \leq p<\infty$ by Başar and Altay [4], and Çolak, et.al. [11]. The paranormed difference sequence space

$$
\Delta \lambda(p)=\left\{x=\left(x_{k}\right) \in w:\left(x_{k}-x_{k-1}\right) \in \lambda(p)\right\},
$$

was investigated by Ahmad and Mursaleen [1] and Malkowsky [24], where $\lambda(p)$ is any of the paranormed spaces $l_{\infty}(p), c(p)$ and $c_{0}(p)$ defined by Simons [27] and Maddox [23].

Many mathematician used Fibonacci matrix defined by numbers of Fibonacci Sequence to construct new sequence spaces. Therefore, firstly, let us look at historical information about Fibonacci Sequence. Fibonacci Sequence consist of $\left\{f_{n}\right\}$ numbers such that each its term is the sum of two terms preceding its. In this sequence, the first two terms are 1 . If we write it clearly, it is a sequence of numbers $1,1,2,3,5,8,13, \ldots$. We can describe it by the equation $f_{n}=f_{n-1}+f_{n-2}$, where $n \geq 2$ and $f_{1}=f_{0}=1$. Fibonacci numbers were come out by Leonardo Pisano Bogollo (c-1170-c1250), he is known with his nickname Fibonacci. Numbers of the sequence is seen in the book "Liber Abaci "firstly written by Leonardo of Pisa. He helped to replace Roman numerical system with the numbers system used today consists of numbers from 0 to 9 in Europa. Fibonacci sequence has some well-known properties such as Golden Ratio and Cassini Formula. If we take ratio of two successive terms of Fibonacci sequence, limit of the this ratio is famous Golden Ratio which is 1.61803 and written by $\phi$.

$$
\begin{gathered}
\lim _{n \rightarrow \infty} \frac{f_{n+1}}{f_{n}}=\frac{1+\sqrt{5}}{2}=\phi \quad \text { (Golden Ratio) } \\
\sum_{k=0}^{n} f_{k}=f_{n+2}-1 \quad \text { for each } n \in \mathbb{N} \\
\sum_{k} \frac{1}{f_{k}} \text { converges. } \\
f_{n-1} \cdot f_{n+1}-f_{n}^{2}=(-1)^{n+1} \text { for each } n \geq 1 \quad \text { (Cassini Formula). }
\end{gathered}
$$


Now, Let's look at definition of aforesaid matrix. Let $f_{n}$ be the $n$-th Fibonacci number for each $n \in \mathbb{N}$. Then, the Fibonacci matrix $\widehat{F}=\left\{\widehat{f_{n k}}\right\}$ is defined as

$$
\widehat{f_{n k}}=\left\{\begin{array}{cll}
\frac{f_{n}}{f_{n+1}} & , \quad(k=n), \\
-\frac{f_{n}}{f_{n+1}} & , \quad(k=n-1), \\
0 & , \quad(0 \leq k<n-1 \quad \text { or } k>n),
\end{array}\right.
$$

for each $k, n \in \mathbb{N}$. Define the sequence $y=\left(y_{n}\right)$ by the $\widehat{F}$ transform of a sequence $x=\left(x_{n}\right)$, i.e.,

$$
y_{n}=\widehat{F}_{n}(x)= \begin{cases}\frac{f_{0}}{f_{1}} x_{0}, & (n=0) \\ \frac{f_{n}}{f_{n+1}} x_{n}-\frac{f_{n+1}}{f_{n}} x_{n-1,}, & (n \geq 1)\end{cases}
$$

for all $n \in \mathbb{N}$. Here are some studies in which used Fibonacci matrix: Kara [14] defined $\ell_{p}(\widehat{F})$ and $\ell_{\infty}(\widehat{F})$ sequence spaces as follows:

$$
l_{p}(f)=\left\{x=\left(x_{n}\right) \in w: \sum_{n}\left|\frac{f_{n}}{f_{n+1}} x_{n}-\frac{f_{n+1}}{f_{n}} x_{n-1}\right|^{p}<\infty\right\}, 1 \leq p<\infty,
$$

and

$$
l_{\infty}(f)=\left\{x=\left(x_{n}\right) \in w: \sup _{n \in \mathbb{N}}\left|\frac{f_{n}}{f_{n+1}} x_{n}-\frac{f_{n+1}}{f_{n}} x_{n-1}\right|<\infty\right\} .
$$

After Kara et al. [15] characterized some class of compact operators on the spaces $\ell_{p}(\widehat{F})$ and $\ell_{\infty}(\widehat{F})$, where $1 \leq p \leq \infty$. Also, Başarır et al. [5] introduced the sequence space $\lambda(\widehat{F})$ and $\mu(\widehat{F}, p)$. In [9] was introduced the generalized Riesz difference sequence space $r^{q}\left(F_{u}^{p}\right)$. Later, Candan [6] presented the sequence spaces $c_{0}(\widehat{F}(r, s))$ and $c(\widehat{F}(r, s))$. Where $\widehat{F}(r, s)$ is the double generalized band matrix $\widehat{F}(r, s)=\left\{f_{n k}(r, s)\right\}$ defined by the sequence $\left(f_{n}\right)$ of Fibonacci numbers as follows:

$$
f_{n k}(r, s)= \begin{cases}s \frac{f_{n+1}}{f_{n}}, & k=n-1, \\ r \frac{f_{n}}{f_{n+1}}, & k=n, \\ 0, & 0 \leq k<n-1 \quad \text { or } \quad k>n\end{cases}
$$

for all $k, n \in \mathbb{N}$, where $r, s \in \mathbb{R} \backslash\{0\}$. After then, Candan and Kayaduman [7] introduced the sequence space $\widehat{c}^{f(r, s)}$ derived by generalized difference Fibonacci matrix. Finally, Candan and Kara [8] investigated the space $\ell_{p}(\widehat{F}(r, s))$, where $1 \leq p \leq \infty$. Recently, [26] presented the sequence spaces $l(\widehat{F}, \mathcal{F}, p, u)$ and $l_{\infty}(\widehat{F}, \mathcal{F}, p, u)$ and researched some topological and geometrical features. Where $\mathcal{F}$ is a sequence of modulus functions, $p=\left(p_{k}\right)$ is any bounded sequence of positive real numbers and $u=\left(u_{k}\right)$ is a sequence of strictly positive real numbers. Motivated by 
these studies, we define the Fibonacci difference sequence spaces $l(\widehat{F}(r, s), \mathcal{F}, p, u)$ and $l_{\infty}(\widehat{F}(r, s), \mathcal{F}, p, u)$ and under the domain of the matrix constituted by using Fibonacci sequence and non-zero real numbers $\mathrm{r}, \mathrm{s}$ and investigate some properties of them. Here are these spaces :

$l(\widehat{F}(r, s), \mathcal{F}, p, u)=\left\{x=\left(x_{k}\right) \in w: \sum_{k}\left[u_{k} F_{k}\left(\left|r \frac{f_{k}}{f_{k+1}} x_{k}+s \frac{f_{k+1}}{f_{k}} x_{k-1}\right|\right)\right]^{p_{k}}<\infty\right\}$,

and

$l_{\infty}(\widehat{F}(r, s), \mathcal{F}, p, u)=\left\{x=\left(x_{k}\right) \in w: \sup _{k \in \mathbb{N}}\left[u_{k} F_{k}\left(\left|r \frac{f_{k}}{f_{k+1}} x_{k}+s \frac{f_{k+1}}{f_{k}} x_{k-1}\right|\right)\right]^{p_{k}}<\infty\right\}$.

We note that the matrix $\widehat{F}(r, s)$ can be reduced to Fibonacci matrix $\widehat{F}$, in case $r=1$ and $s=-1$. Therefore the results related to domain of the matrix $\widehat{F}(r, s)$ are more general and across the board than those of the matrix domain of $\widehat{F}$ and include them.

Now, let us give definition of modulus function which we used in this paper. A modulus function is a function $f:[0, \infty) \rightarrow[0, \infty)$ such that:

i) $f(x)=0$ if and only if $x=0$,

ii) $f(x+y) \leq f(x)+f(y)$, for all $x, y \geq 0$,

iii) $f$ increasing,

iv) $f$ is continuous from the right at 0 .

We can say that $f$ must be continuous everywhere on $[0, \infty)$. The function may be bounded or unbounded. For instance, The modulus function $f(x)=\frac{x}{x+1}$ is bounded, but the function $f(x)=x^{p}, 0<p<1$ is unbounded.

We will need to following datas in our calculating:

$$
f_{n k}^{-1}(r, s)=\left\{\begin{array}{cc}
\frac{1}{r}\left(-\frac{s}{r}\right)^{n-k} \frac{f_{n+1}^{2}}{f_{k} f_{k+1}}, & 0 \leq k \leq n \\
0, & k \geq n
\end{array}\right.
$$

Additionaly, specify the sequence $y=\left(y_{n}\right)$ by the $\widehat{F}(r, s)$-transform of a sequence $x=\left(x_{n}\right)$, i.e.

$$
y_{n}=(\widehat{F}(r, s)(x))_{n}= \begin{cases}r x_{0}, & n=0 \\ r \frac{f_{n}}{f_{n+1}} x_{n}+s \frac{f_{n+1}}{f_{n}} x_{n-1}, & n \geq 1\end{cases}
$$

The following inequality will be used throughout the study. Let $p=\left(p_{k}\right)$ be sequence of positive real numbers with $0<p_{k} \leq \sup _{k} p_{k}=H$ and let $D=$ $\max \left\{1,2^{H-1}\right\}$. Then for factorable sequences $\left(a_{k}\right)$ and $\left(b_{k}\right)$ in the complex plane, we have

$$
\left|a_{k}+b_{k}\right|^{p_{k}} \leq D\left(\left|a_{k}\right|^{p_{k}}+\left|b_{k}\right|^{p_{k}}\right)
$$


Also, we assume throughout the study that $p, q \geq 1$ with $\frac{1}{p}+\frac{1}{q}=1$ and denote the collection of all finite subsets of $\mathbb{N}$ by $\mathcal{H}$.

In this study, we gave some algebraic and topological features of the sequence spaces $l(\widehat{F}(r, s), \mathcal{F}, p, u)$ and $l_{\infty}(\widehat{F}(r, s), \mathcal{F}, p, u)$ in section 2. In section 3, $\alpha-, \beta-, \gamma$-duals of these spaces were acquired. In section 4 , we characterized some matrix classes on the these sequence spaces and finally, some geometric features of the space $l(\widehat{F}(r, s), \mathcal{F}, p, u)$ were given.

\section{Some Algebraic and Topological Properties of The Spaces$$
l(\widehat{F}(r, s), \mathcal{F}, p, u) \text { and } l_{\infty}(\widehat{F}(r, s), \mathcal{F}, p, u)
$$

Theorem 2.1. $l(\widehat{F}(r, s), \mathcal{F}, p, u)$ and $l_{\infty}(\widehat{F}(r, s), \mathcal{F}, p, u)$ are linear spaces over complex field $\mathbb{C}$.

Proof. Let $x, y \in l(\widehat{F}(r, s), \mathcal{F}, p, u)$. Then

$$
\sum_{k}\left[u_{k} F_{k}\left(\left|\left(r \frac{f_{k}}{f_{k+1}} x_{k}+s \frac{f_{k+1}}{f_{k}} x_{k-1}\right)\right|\right)\right]^{p_{k}}<\infty
$$

and

$$
\sum_{k}\left[u_{k} F_{k}\left(\left|\left(r \frac{f_{k}}{f_{k+1}} y_{k}+s \frac{f_{k+1}}{f_{k}} y_{k-1}\right)\right|\right)\right]^{p_{k}}<\infty
$$

For $\lambda, \mu \in \mathbb{C}$, there exist integers $M_{\lambda}, N_{\mu}$ such that $|\lambda| \leq M_{\lambda}$ and $|\mu| \leq N_{\mu}$. Using inequality 1.2 and definition of modulus function, we have

$$
\begin{aligned}
& \sum_{k}\left[u_{k} F_{k}\left(\left|\lambda\left(r \frac{f_{k}}{f_{k+1}} x_{k}+s \frac{f_{k+1}}{f_{k}} x_{k-1}\right)+\mu\left(r \frac{f_{k}}{f_{k+1}} y_{k}+s \frac{f_{k+1}}{f_{k}} y_{k-1}\right)\right|\right)\right]^{p_{k}} \\
\leq & \sum_{k}\left[u_{k} F_{k}\left(|\lambda|\left|\left(r \frac{f_{k}}{f_{k+1}} x_{k}+s \frac{f_{k+1}}{f_{k}} x_{k-1}\right)\right|\right)\right]^{p_{k}} \\
& +\sum_{k}\left[u_{k} F_{k}\left(|\mu|\left|\left(r \frac{f_{k}}{f_{k+1}} y_{k}+s \frac{f_{k+1}}{f_{k}} y_{k-1}\right)\right|\right)\right]^{p_{k}} \\
\leq & D M_{\lambda}^{H} \sum_{k}\left[u_{k} F_{k}\left(\left|\left(r \frac{f_{k}}{f_{k+1}} x_{k}+s \frac{f_{k+1}}{f_{k}} x_{k-1}\right)\right|\right)\right]^{p_{k}} \\
& +D N_{\mu}^{H} \sum_{k}\left[u_{k} F_{k}\left(\left|\left(r \frac{f_{k}}{f_{k+1}} y_{k}+s \frac{f_{k+1}}{f_{k}} y_{k-1}\right)\right|\right)\right]^{p_{k}} \\
< & \infty
\end{aligned}
$$


Thus $\lambda x+\mu y \in l(\widehat{F}(r, s), \mathcal{F}, p, u)$. So, proof is completed. The same way, we can obtain that $l_{\infty}(\widehat{F}(r, s), \mathcal{F}, p, u)$ is a linear space.

Theorem 2.2. $l(\widehat{F}(r, s), \mathcal{F}, p, u)$ is paranormed space with

$$
g(x)=\sup _{k}\left(\sum_{k}\left[u_{k} F_{k}\left(\left|\left(r \frac{f_{k}}{f_{k+1}} x_{k}+s \frac{f_{k+1}}{f_{k}} x_{k-1}\right)\right|\right)\right]^{p_{k}}\right)^{\frac{1}{K}}
$$

where $0<p_{k} \leq \sup _{k} p_{k}=H<\infty$ and $K=\max \{1, H\}$.

Proof. For all $x \in l(\widehat{F}(r, s), \mathcal{F}, p, u)$, It is trivial that $g(x)=g(-x)$. Also we can see easily that $r \frac{f_{k}}{f_{k+1}} x_{k}+s \frac{f_{k+1}}{f_{k}} x_{k-1}=0$, for $x=0$. Since $\frac{p_{k}}{K} \leq 1$, using Minkowsky Inequality, we have

$$
\begin{gathered}
\left(\sum_{k}\left[u_{k} F_{k}\left(\left|\left(r \frac{f_{k}}{f_{k+1}} x_{k}+s \frac{f_{k+1}}{f_{k}} x_{k-1}\right)+\left(r \frac{f_{k}}{f_{k+1}} y_{k}+s \frac{f_{k+1}}{f_{k}} y_{k-1}\right)\right|\right)\right]^{p_{k}}\right)^{\frac{1}{K}} \\
\leq\left(\sum_{k}\left[u_{k} F_{k}\left(\left|\left(r \frac{f_{k}}{f_{k+1}} x_{k}+s \frac{f_{k+1}}{f_{k}} x_{k-1}\right)\right|\right)+u_{k} F_{k}\left(\left|r \frac{f_{k}}{f_{k+1}} y_{k}+s \frac{f_{k+1}}{f_{k}} y_{k-1}\right|\right)\right]^{p_{k}}\right)^{\frac{1}{K}} \\
\leq\left(\sum_{k}\left[u_{k} F_{k}\left(\left|\left(r \frac{f_{k}}{f_{k+1}} x_{k}+s \frac{f_{k+1}}{f_{k}} x_{k-1}\right)\right|\right)\right]^{p_{k}}\right)^{\frac{1}{K}} \\
+\left(\sum_{k}\left[u_{k} F_{k}\left(\left|r \frac{f_{k}}{f_{k+1}} y_{k}+s \frac{f_{k+1}}{f_{k}} y_{k-1}\right|\right)\right]^{p_{k}}\right)^{\frac{1}{K}} .
\end{gathered}
$$

Therefore $g(x)$ is subadditive. For the continuity of multiplication, let us take any complex number $\alpha$. By definition, we have

$$
\begin{aligned}
g(\alpha x) & =\sup _{k}\left(\sum_{k}\left[u_{k} F_{k}\left(\left|\alpha\left(r \frac{f_{k}}{f_{k+1}} x_{k}+s \frac{f_{k+1}}{f_{k}} x_{k-1}\right)\right|\right)\right]^{p_{k}}\right)^{\frac{1}{K}} \\
& \leq C_{\alpha}^{\frac{H}{K}} g(x)
\end{aligned}
$$

where $C_{\alpha}$ is a positive integer such that $|\alpha| \leq C_{\alpha}$. Now, let $\alpha \rightarrow 0$ for any fixed $x$ with $g(x)=0$. From definition, taking small enough $\alpha$, for $|\alpha|<1$ and $1 \leq n<n_{0}$, we get

$$
\sum_{k}\left[u_{k} F_{k}\left(\left|\left(r \frac{f_{k}}{f_{k+1}} x_{k}+s \frac{f_{k+1}}{f_{k}} x_{k-1}\right)\right|\right)\right]^{p_{k}}<\varepsilon, \text { for } n>n_{0}(\varepsilon)
$$

Since $F_{k}$ is continuous, we get

$$
\sum_{k}\left[u_{k} F_{k}\left(\left|\left(r \frac{f_{k}}{f_{k+1}} x_{k}+s \frac{f_{k+1}}{f_{k}} x_{k-1}\right)\right|\right)\right]^{p_{k}}<\varepsilon
$$


From equation 2.1 and 2.2 , we have

$$
g(\alpha x) \rightarrow 0 \text { as } \alpha \rightarrow 0 .
$$

This completes the proof.

Theorem 2.3. If $p=\left(p_{k}\right)$ and $q=\left(q_{k}\right)$ are bounded sequences of positive real numbers with $0 \leq p_{k} \leq q_{k}<\infty$ for each $k$, then

$$
l(\widehat{F}(r, s), \mathcal{F}, p, u) \subseteq l(\widehat{F}(r, s), \mathcal{F}, q, u) .
$$

Proof. Let $x \in l(\widehat{F}(r, s), \mathcal{F}, p, u)$. Then

$$
\sum_{k}\left[u_{k} F_{k}\left(\left|\left(r \frac{f_{k}}{f_{k+1}} x_{k}+s \frac{f_{k+1}}{f_{k}} x_{k-1}\right)\right|\right)\right]^{p_{k}}<\infty .
$$

This implies that

$$
\sum_{k}\left[u_{k} F_{k}\left(\left|\left(r \frac{f_{k}}{f_{k+1}} x_{k}+s \frac{f_{k+1}}{f_{k}} x_{k-1}\right)\right|\right)\right]^{p_{k}} \leq 1 .
$$

For sufficiently large values of $k$ (say) $k \geq k_{0}$, for some fixed $k_{0} \in \mathbb{N}$, since $F_{k}$ is increasing and $p_{k} \leq q_{k}$, we get a sequence

$$
\begin{aligned}
\sum_{k \geq k_{0}}\left[u_{k} F_{k}\right. & \left.\left(\left|\left(r \frac{f_{k}}{f_{k+1}} x_{k}+s \frac{f_{k+1}}{f_{k}} x_{k-1}\right)\right|\right)\right]^{q_{k}} \\
& \leq \sum_{k \geq k_{0}}\left[u_{k} F_{k}\left(\left|\left(r \frac{f_{k}}{f_{k+1}} x_{k}+s \frac{f_{k+1}}{f_{k}} x_{k-1}\right)\right|\right)\right]^{p_{k}}<\infty .
\end{aligned}
$$

Hence $x \in l(\widehat{F}(r, s), \mathcal{F}, q, u)$. This completes the proof.

Theorem 2.4. Let $\mathcal{F}=\left(F_{k}\right)$ be a sequence of modulus functions and $\beta=\lim _{t \rightarrow \infty} \frac{F_{k}(t)}{t}>0$. Then

$$
l(\widehat{F}(r, s), \mathcal{F}, p, u) \subseteq l(\widehat{F}(r, s), p, u) .
$$

Proof. To prove, let us take $\beta>0$. From the definition of $\beta$, we have $F_{k}(t) \geq \beta(t)$, and $t \leq \frac{1}{\beta} F_{k}(t)$ for all $t>0$. Let us take a sequence

$$
x=\left(x_{k}\right) \in l(\widehat{F}(r, s), \mathcal{F}, p, u) .
$$

Then we have

$$
\begin{aligned}
\sum_{k}\left[u_{k}\left(\left|\left(r \frac{f_{k}}{f_{k+1}} x_{k}+s \frac{f_{k+1}}{f_{k}} x_{k-1}\right)\right|\right)\right]^{p_{k}} & \\
& \leq \frac{1}{\beta} \sum_{k}\left[u_{k} F_{k}\left(\left|\left(r \frac{f_{k}}{f_{k+1}} x_{k}+s \frac{f_{k+1}}{f_{k}} x_{k-1}\right)\right|\right)\right]^{p_{k}},
\end{aligned}
$$

which implies that $x=\left(x_{k}\right) \in l(\widehat{F}(r, s), p, u)$.This completes the proof. 
Theorem 2.5. Let $\mathcal{F}^{1}=\left(F_{k}^{1}\right)$ and $\mathcal{F}^{2}=\left(F_{k}^{2}\right)$ are sequences of modulus functions, then

$$
l\left(\widehat{F}(r, s), \mathcal{F}^{1}, p, u\right) \cap l\left(\widehat{F}(r, s), \mathcal{F}^{2}, p, u\right) \subseteq l\left(\widehat{F}(r, s), \mathcal{F}^{1}+\mathcal{F}^{2}, p, u\right) .
$$

Proof. $x=\left(x_{k}\right) \in l\left(\widehat{F}(r, s), \mathcal{F}^{1}, p, u\right) \cap l\left(\widehat{F}(r, s), \mathcal{F}^{2}, p, u\right)$. Therefore

$$
\sum_{k}\left[u_{k} F_{k}^{1}\left(\left|\left(r \frac{f_{k}}{f_{k+1}} x_{k}+s \frac{f_{k+1}}{f_{k}} x_{k-1}\right)\right|\right)\right]^{p_{k}}<\infty
$$

and

$$
\sum_{k}\left[u_{k} F_{k}^{2}\left(\left|\left(r \frac{f_{k}}{f_{k+1}} x_{k}+s \frac{f_{k+1}}{f_{k}} x_{k-1}\right)\right|\right)\right]^{p_{k}}<\infty
$$

Then, we have

$$
\begin{aligned}
& \sum_{k}\left[u_{k}\left(F_{k}^{1}+F_{k}^{2}\right)\left(\left|\left(r \frac{f_{k}}{f_{k+1}} x_{k}+s \frac{f_{k+1}}{f_{k}} x_{k-1}\right)\right|\right)\right]^{p_{k}} \\
\leq & K\left\{\sum_{k}\left[u_{k} F_{k}^{1}\left(\left|\left(r \frac{f_{k}}{f_{k+1}} x_{k}+s \frac{f_{k+1}}{f_{k}} x_{k-1}\right)\right|\right)\right]^{p_{k}}\right\} \\
& +K\left\{\sum_{k}\left[u_{k} F_{k}^{2}\left(\left|\left(r \frac{f_{k}}{f_{k+1}} x_{k}+s \frac{f_{k+1}}{f_{k}} x_{k-1}\right)\right|\right)\right]^{p_{k}}\right\} .
\end{aligned}
$$

Thus

$$
\sum_{k}\left[u_{k}\left(F_{k}^{1}+F_{k}^{2}\right)\left(\left|\left(r \frac{f_{k}}{f_{k+1}} x_{k}+s \frac{f_{k+1}}{f_{k}} x_{k-1}\right)\right|\right)\right]^{p_{k}}<\infty .
$$

That is $x=\left(x_{k}\right) \in l\left(\widehat{F}(r, s), \mathcal{F}^{1}+\mathcal{F}^{2}, p, u\right)$ and this completes the proof.

Theorem 2.6. Let $1 \leq p_{k} \leq H \leq \infty$ for all $k \in \mathbb{N}$. Then $l(\widehat{F}(r, s), \mathcal{F}, p, u)$ and $l_{\infty}(\widehat{F}(r, s), \mathcal{F}, p, u)$ are normed spaces with the norms

$$
\|x\|_{l(\widehat{F}(r, s), \mathcal{F}, p, u)}=\left(\sum_{n}\left[u_{k} F_{k}\left(\left|\widehat{F_{n}}(r, s)(x)\right|\right)\right]^{p_{k}}\right)^{\frac{1}{K}},
$$

and

$$
\|x\|_{l_{\infty}(\widehat{F}(r, s), \mathcal{F}, p, u)}=\sup _{n \in \mathbb{N}}\left[u_{k} F_{k}\left(\left|\widehat{F_{n}}(r, s)(x)\right|\right)\right]^{p_{k}}
$$

respectively. 
Proof. It can be verified easily. Therefore we omit the proof.

Remark 2.1. It can be seen easily that the absolute property does not provide on the spaces $l(\widehat{F}(r, s), \mathcal{F}, p, u)$ and $l_{\infty}(\widehat{F}(r, s), \mathcal{F}, p, u)$, i.e.,

$$
\|x\|_{l(\widehat{F}(r, s), \mathcal{F}, p, u)} \neq\|\mid x\|_{l(\widehat{F}(r, s), \mathcal{F}, p, u)}
$$

and

$$
\|x\|_{l_{\infty}(\widehat{F}(r, s), \mathcal{F}, p, u)} \neq\||x|\|_{l_{\infty}(\widehat{F}(r, s), \mathcal{F}, p, u)}
$$

for at least one sequence in both the spaces $l(\widehat{F}(r, s), \mathcal{F}, p, u)$ and $l_{\infty}(\widehat{F}(r, s), \mathcal{F}, p, u)$; this illustrates that $l(\widehat{F}(r, s), \mathcal{F}, p, u)$ and $l_{\infty}(\widehat{F}(r, s), \mathcal{F}, p, u)$ are the sequence spaces of non-absolute type, in which $|x|=\left(\left|x_{k}\right|\right)$ and $1 \leq p_{k} \leq H<\infty$ for all $k \in \mathbb{N}$.

Theorem 2.7. The sequence spaces $l(\widehat{F}(r, s), \mathcal{F}, p, u)$ of non absolute type is linearly isomorphic to the space $l_{p}$, for $1 \leq p_{k} \leq H<\infty$ for all $k \in \mathbb{N}$.

Proof. To exhibit that the existence of a linear bijection between the spaces $l(\widehat{F}(r, s), \mathcal{F}, p, u)$ and $l_{p}$ for $1 \leq p_{k} \leq H<\infty$ for all $k \in \mathbb{N}$. Take into account the transformation $T$ defined with the notation 1.1 , from $l(\widehat{F}(r, s), \mathcal{F}, p, u)$ to $l_{p}$ by $x \rightarrow y=T x$. Then $T x=y=\widehat{F}(r, s) x \in l_{p}$, for $x \in l(\widehat{F}(r, s), \mathcal{F}, p, u)$. Also, the linearity of $T$ is clear. In addition, it is very easy that $x=0$ whenever $T x=0$ and therefore $T$ is injective. We consider that $y=\left(y_{k}\right) \in l_{p}$, for $1 \leq p_{k} \leq H<\infty$ for all $k \in \mathbb{N}$ and define sequence $x=\left(x_{k}\right)$ by

$$
x_{k}=\sum_{j=0}^{k}\left(\frac{1}{r}\right)\left(-\frac{s}{r}\right)^{k-j} \frac{f_{k+1}^{2}}{f_{j} f_{j+1}} y_{j}, \mathrm{k} \in \mathbb{N} .
$$

Then, in the case $1 \leq p_{k} \leq H<\infty$, for all $k \in \mathbb{N}$ and $p=\infty$, we get

$$
\begin{aligned}
\|x\|_{l(\widehat{F}(r, s), \mathcal{F}, p, u)} & =\left(\sum_{k}\left[u_{k} F_{k}\left(\left|r \frac{f_{k}}{f_{k+1}} x_{k}+s \frac{f_{k+1}}{f_{k}} x_{k-1}\right|\right)\right]^{p_{k}}\right)^{\frac{1}{K}} \\
& =\left(\sum _ { k } \left[u _ { k } F _ { k } \left(\mid r \frac{f_{k}}{f_{k+1}} \sum_{j=0}^{k}\left(\frac{1}{r}\right)\left(-\frac{s}{r}\right)^{k-j} \frac{f_{k+1}^{2}}{f_{j} f_{j+1}} y_{j}\right.\right.\right. \\
& \left.\left.\left.+s \frac{f_{k+1}}{f_{k}} \sum_{j=0}^{k-1}\left(\frac{1}{r}\right)\left(-\frac{s}{r}\right)^{k-j} \cdot \frac{f_{k}}{f_{j} f_{j+1}} y_{j} \mid\right)\right]^{p_{k}}\right)^{\frac{1}{K}} \\
& =\left(\sum_{k}\left|y_{k}\right|^{p_{k}}\right)^{\frac{1}{K}} \\
& =\|y\|_{p}<\infty
\end{aligned}
$$


and

$$
\|x\|_{l_{\infty}(\widehat{F}(r, s), \mathcal{F}, p, u)}=\sup _{k \in \mathbb{N}}\left[u_{k} F_{k}\left(\left|\widehat{F}_{k}(r, s)(x)\right|\right)\right]^{p_{k}}=\|y\|_{\infty}<\infty
$$

respectively. Therefore $T$ is linear bijection which means that the spaces $l_{p}$ and $l(\widehat{F}(r, s), \mathcal{F}, p, u)$ are linearly isomorphic for $1 \leq p_{k} \leq H<\infty$ for all $k \in \mathbb{N}$.

3. The $\alpha-, \beta-, \gamma-$ Duals of the space $l(\widehat{F}(r, s), \mathcal{F}, p, u)$ The $\alpha-, \beta-, \gamma-$ duals of the sequence space $X$ are defined by

$$
\begin{aligned}
& X^{\alpha}=\left\{a=\left(a_{k}\right) \in w: a x=\left(a_{k} x_{k}\right) \in l_{1} \text { for all } x=\left(x_{k}\right) \in X\right\}, \\
& X^{\beta}=\left\{a=\left(a_{k}\right) \in w: a x=\left(a_{k} x_{k}\right) \in c s \text { for all } x=\left(x_{k}\right) \in X\right\},
\end{aligned}
$$

and

$$
X^{\gamma}=\left\{a=\left(a_{k}\right) \in w: a x=\left(a_{k} x_{k}\right) \in b s \text { for all } x=\left(x_{k}\right) \in X\right\},
$$

respectively. Where $c s$ and $b s$ are the sequence spaces of all convergent and bounded series, respectively. In [28], The following known results are vital for our investigation.

Lemma 3.1. $A=\left(a_{n k}\right) \in\left(l_{p}, l_{1}\right)$ iff

$$
\sup _{K \in \mathcal{H}} \sum_{k}\left|\sum_{n \in K} a_{n k}\right|<\infty, 1<p \leq \infty .
$$

Lemma 3.2. $A=\left(a_{n k}\right) \in\left(l_{p}, c\right)$ iff

$$
\lim _{n \rightarrow \infty} \text { ank exists for all } k \in \mathbb{N}
$$

$$
\sup _{n \in \mathbb{N}} \sum_{k}\left|a_{n k}\right|^{q}<\infty, \quad 1<p<\infty
$$

Lemma 3.3. $A=\left(a_{n k}\right) \in\left(l_{\infty}, c\right)$ iff

$$
\lim _{n \rightarrow \infty} \text { ank exists for all } k \in \mathbb{N}
$$

and

$$
\lim _{n \rightarrow \infty} \sum_{k}\left|a_{n k}\right|=\sum_{k}\left|\lim _{n \rightarrow \infty} a_{n k}\right| .
$$


Lemma 3.4. $A=\left(a_{n k}\right) \in\left(l_{p}, l_{\infty}\right)$ iff

$$
\sup _{n \in \mathbb{N}} \sum_{k}\left|a_{n k}\right|^{q}<\infty, \quad 1<p<\infty
$$

holds with $1<p \leq \infty$.

Theorem 3.1. The $\alpha$-dual of the space $l(\widehat{F}(r, s), \mathcal{F}, p, u)$ is the set

$d_{1}=\left\{a=\left(a_{k}\right) \in w: \sup _{K \in \mathcal{H}} \sum_{k}\left[u_{k} F_{k}\left(\left|\sum_{n \in K}\left(\frac{1}{r}\right)\left(-\frac{s}{r}\right)^{n-k} \frac{f_{n+1}^{2}}{f_{k} f_{k+1}} a_{n}\right|^{q}\right)\right]^{p_{k}}<\infty\right\}$,

where $1<p \leq \infty$, for all $k \in \mathbb{N}$.

Proof. For all $k \in \mathbb{N}, 1<p_{k} \leq H<\infty$ and for any fixed sequence $a=\left(a_{n}\right) \in w$, we define the matrix $B=\left(b_{n k}\right)$ by

$$
b_{n k}= \begin{cases}\sum_{k}\left[u_{k} F_{k}\left(\left|\left(\frac{1}{r}\right)\left(-\frac{s}{r}\right)^{n-k} \frac{f_{n+1}^{2}}{f_{k} f_{k+1}} a_{n}\right|\right)\right]^{p_{k}}, & 0 \leq k \leq n, \\ 0, & k>n,\end{cases}
$$

for each $n, k \in \mathbb{N}$. Also for every $x=\left(x_{n}\right) \in w$, we put $y=\widehat{F}(r, s)(x)$. Then it follows by 1.1 that

$$
a_{n} x_{n}=\sum_{k}\left[u_{k} F_{k}\left(\left|\sum_{k=0}^{n}\left(\frac{1}{r}\right)\left(-\frac{s}{r}\right)^{n-k} \frac{f_{n+1}^{2}}{f_{k} f_{k+1}} a_{n} y_{k}\right|\right)\right]^{p_{k}}=B_{n}(y),
$$

$n \in \mathbb{N}$. Therefore, we derive by using Lemma 3.1 that

$$
\sup _{K \in \mathcal{H}} \sum_{k}\left[u_{k} F_{k}\left(\left|\sum_{k=0}^{n}\left(\frac{1}{r}\right)\left(-\frac{s}{r}\right)^{n-k} \frac{f_{n+1}^{2}}{f_{k} f_{k+1}} a_{n} y_{k}\right|^{q}\right)\right]^{p_{k}}<\infty
$$

which implies that $l(\widehat{F}(r, s), \mathcal{F}, p, u)^{\alpha}=d_{1}$.

Theorem 3.2. Define the sets $d_{2}, d_{3}, d_{4}$ by

$$
\begin{aligned}
& d_{2}=\left\{a=\left(a_{k}\right) \in w: \sum_{k}\left[u_{k} F_{k}\left(\left|\sum_{j=k}^{\infty}\left(\frac{1}{r}\right)\left(-\frac{s}{r}\right)^{n-k} \frac{f_{j+1}^{2}}{f_{k} f_{k+1}} a_{j}\right|\right)\right]^{p_{k}} \text { exists for all } k \in \mathbb{N}\right\}, \\
& d_{3}=\left\{a=\left(a_{k}\right) \in w: \sup _{n \in \mathbb{N}} \sum_{k=0}^{n}\left[u_{k} F_{k}\left(\left|\sum_{j=k}^{n}\left(\frac{1}{r}\right)\left(-\frac{s}{r}\right)^{n-k} \frac{f_{j+1}^{2}}{f_{k} f_{k+1}} a_{j}\right|^{q}\right)\right]^{p_{k}}<\infty\right\},
\end{aligned}
$$




$$
\begin{array}{r}
d_{4}=\left\{a=\left(a_{k}\right) \in w\right. \\
: \lim _{n \rightarrow \infty} \sum_{k=0}^{n}\left[u_{k} F_{k}\left(\left|\sum_{j=k}^{n}\left(\frac{1}{r}\right)\left(-\frac{s}{r}\right)^{n-k} \frac{f_{j+1}^{2}}{f_{k} f_{k+1}} a_{j}\right|\right)\right]^{p_{k}} \\
\left.=\sum_{k}\left[u_{k} F_{k}\left(\left|\sum_{j=k}^{\infty}\left(\frac{1}{r}\right)\left(-\frac{s}{r}\right)^{n-k} \frac{f_{j+1}^{2}}{f_{k} f_{k+1}} a_{j}\right|\right)\right]^{p_{k}}<\infty\right\}
\end{array}
$$

Then $[l(\widehat{F}(r, s), \mathcal{F}, p, u)]^{\beta}=d_{2} \cap d_{3}$ and $\left[l_{\infty}(\widehat{F}(r, s), \mathcal{F}, p, u)\right]^{\beta}=d_{2} \cap d_{4}$, where $1 \leq p_{k} \leq H=\sup _{k} p_{k}<\infty$, for all $k \in \mathbb{N}$.

Proof. Let $a=\left(a_{k}\right) \in w$ and consider the equality

$$
\begin{aligned}
\sum_{k=0}^{n} a_{k} x_{k} & =\sum_{k=0}^{n} a_{k}\left(\sum_{j=0}^{n} u_{j} F j\left(\left|\left(\frac{1}{r}\right)\left(-\frac{s}{r}\right)^{k-j} \frac{f_{k+1}^{2}}{f_{j} f_{j+1}} y_{j}\right|\right)\right) \\
& =\sum_{k=0}^{n}\left(\sum_{j=k}^{n}\left(\frac{1}{r}\right)\left(-\frac{s}{r}\right)^{j-k} \frac{f_{j+1}^{2}}{f_{k} f_{k+1}} a_{j}\right) y_{k}=D_{n}(y)
\end{aligned}
$$

Where $D=\left(d_{n k}\right)$ is defined by

$$
d_{n k}= \begin{cases}\sum_{j=k}^{n}\left(\frac{1}{r}\right)\left(-\frac{s}{r}\right)^{j-k} \frac{f_{j+1}^{2}}{f_{k} f_{k+1}} a_{j}, & 0 \leq k \leq n \\ 0, & k>n,\end{cases}
$$

where $n, k \in \mathbb{N}$.Then we deduce from Lemma 3.2 with 1.1 that $a x=\left(a_{k} x_{k}\right) \in c s$ whenever $x=\left(x_{k}\right) \in l(\widehat{F}(r, s), \mathcal{F}, p, u)$ iff $D y \in c$ whenever $y=\left(y_{k}\right) \in l_{p}$. Thus $\left(a_{k}\right) \in l(\widehat{F}(r, s), \mathcal{F}, p, u)$ iff $\left(a_{k}\right) \in d_{2}$ and $\left(a_{k}\right) \in d_{3}$ by 3.1 and 3.2, respectively. Hence $l(\widehat{F}(r, s), \mathcal{F}, p, u)^{\beta}=d_{2} \cap d_{3}$. It is clear that one can also prove the case $p=\infty$ by the technique used in the proof of the case $1<p<\infty$ with Lemma 3.3 instead of Lemma 3.2. So we leave the detailed proof to the reader.

Theorem 3.3. $l(\widehat{F}(r, s), \mathcal{F}, p, u)^{\gamma}=d_{3}$, where $1<p_{k} \leq H \leq \infty$ for all $k \in \mathbb{N}$.

Proof. The result can be obtained from Lemma 3.4. 


\section{Some Matrix Transformations}

In this chapter, we characterize the classes $(l(\widehat{F}(r, s), \mathcal{F}, p, u), X)$, where $1<p_{k} \leq H<\infty$, for all $k \in \mathbb{N}$ and $X$ is any of the spaces $l_{\infty}, l_{1}, c$ and $c_{0}$. For simplicity in notation, we write

$$
\widetilde{a}_{n k}=\sum_{k}\left[u_{k} F_{k}\left(\left|\sum_{j=k}^{\infty}\left(\frac{1}{r}\right)\left(-\frac{s}{r}\right)^{j-k} \frac{f_{j+1}^{2}}{f_{k} f_{k+1}} a_{n j}\right|\right)\right]^{p_{k}}
$$

for all $k, n \in \mathbb{N}$.

Lemma 4.1. [16] Let $\lambda$ be an FK- space, $U$ be a triangle, $V$ be its inverse and $\mu$ be an arbitrary subset of $w$. Then we have

$A=\left(a_{n k}\right) \in\left(\lambda_{U}, \mu\right)$ iff

$$
C^{(n)}=\left(c_{m k}^{(n)}\right) \in(\lambda, c) \text { for all } n \in \mathbb{N},
$$

and

$$
C=\left(c_{n k}\right) \in(\lambda, \mu),
$$

where

$$
c_{m k}^{(n)}= \begin{cases}\sum_{j=k}^{m} a_{n j} v_{j k}, & 0 \leq k \leq m, \\ 0, & (k>m)\end{cases}
$$

and

$$
c_{n k}=\sum_{j=k}^{\infty} a_{n j} v_{j k}, \text { for all } k, m, n \in \mathbb{N} .
$$

We can write from this lemma that

$$
A \in\left(\lambda_{\widehat{F}(r, s)}, \mu\right) \Leftrightarrow D^{(n)}=\left(d_{m k}^{(n)}\right) \in(\lambda, c) \text { for all } n \in \mathbb{N} \text { and } D=\left(d_{n k}\right) \in(\lambda, \mu)
$$
where

$$
\begin{gathered}
d_{m k}^{(n)}= \begin{cases}\sum_{j=k}^{m} \frac{1}{r}\left(-\frac{s}{r}\right)^{j-k} \frac{f_{j+1}^{2}}{f_{k} f_{k+1}} a_{n j}, & 0 \leq k \leq m, \\
0, & k>m,\end{cases} \\
d_{n k}=\sum_{j=k}^{\infty} \frac{1}{r}\left(-\frac{s}{r}\right)^{j-k} \frac{f_{j+1}^{2}}{f_{k} f_{k+1}} a_{n j}, \text { for all } k, m, n \in \mathbb{N} .
\end{gathered}
$$


Now, we list the following conditions:

$$
\begin{aligned}
& \text { (4.1) } \sup _{m \in \mathbb{N}} \sum_{k=0}^{m}\left[u_{k} F_{k}\left(\left|\sum_{j=k}^{m} \frac{1}{r}\left(-\frac{s}{r}\right)^{j-k} \frac{f_{j+1}^{2}}{f_{k} f_{k+1}} a_{n j}\right|^{q}\right)\right]^{p_{k}}<\infty \\
& \text { (4.2) } \lim _{m \rightarrow \infty}\left[u_{k} F_{k}\left(\left|\sum_{j=k}^{m} \frac{1}{r}\left(-\frac{s}{r}\right)^{j-k} \frac{f_{j+1}^{2}}{f_{k} f_{k+1}} a_{n j}\right|\right)\right]^{p_{k}}=\widetilde{a}_{n k}, \quad \text { for all } n, k \in \mathbb{N}
\end{aligned}
$$

(4.3) $\lim _{m \rightarrow \infty} \sum_{k=0}^{m}\left[u_{k} F_{k}\left(\left|\sum_{j=k}^{m} \frac{1}{r}\left(-\frac{s}{r}\right)^{j-k} \frac{f_{j+1}^{2}}{f_{k} f_{k+1}} a_{n j}\right|\right)\right]^{p_{k}}=\sum_{k}\left|\widetilde{a}_{n k}\right|$, for each $n \in \mathbb{N}$

$$
\begin{aligned}
& \sup _{\mathrm{n} \in \mathbb{N}} \sum_{k}\left|\widetilde{a}_{n k}\right|^{q}<\infty \\
& \sup _{n \in \mathcal{H}} \sum_{k}\left|\sum_{n \in \mathbb{N}} \widetilde{a}_{n k}\right|^{q}<\infty \\
& \lim _{n \rightarrow \infty} \widetilde{a}_{n k}=\widetilde{a}_{k}, \quad \mathrm{k} \in \mathbb{N} \\
& \lim _{n \rightarrow \infty} \sum_{k}\left|\widetilde{a}_{n k}\right|=\sum_{k}\left|\widetilde{a}_{k}\right| \\
& \lim _{n \rightarrow \infty} \sum_{k} \widetilde{a}_{n k}=0 \\
& \sup _{n, k \in \mathbb{N}}\left|\widetilde{a}_{n k}\right|<\infty \\
& \sup _{k, m \in \mathbb{N}}\left[u_{k} F_{k}\left(\left|\sum_{j=k}^{m} \frac{1}{r}\left(-\frac{s}{r}\right)^{j-k} \frac{f_{j+1}^{2}}{f_{k} f_{k+1}} a_{n j}\right|\right)\right]^{p_{k}}<\infty \\
& \sup _{k \in \mathbb{N}} \sum_{k}\left|\widetilde{a}_{n k}\right|<\infty \\
& \sup _{N, K \in \mathcal{H}}\left|\sum_{n \in \mathbb{N}} \sum_{k \in K} \widetilde{a}_{n k}\right|<\infty
\end{aligned}
$$


Theorem 4.1. i) $A=\left(a_{n k}\right) \in\left(l_{1}(\widehat{F}(r, s), \mathcal{F}, p, u), l_{\infty}\right)$ if and only if 4.2, 4.9, 4.10 hold.

ii) $A=\left(a_{n k}\right) \in\left(l_{1}(\widehat{F}(r, s), \mathcal{F}, p, u), c\right)$ if and only if 4.2, 4.6, 4.9, 4.10 hold.

iii) $A=\left(a_{n k}\right) \in\left(l_{1}(\widehat{F}(r, s), \mathcal{F}, p, u), c_{0}\right)$ if and only if 4.2, 4.6 with $\widetilde{a}_{k}=0$,

\section{9, 4.10 hold.}

iv) $A=\left(a_{n k}\right) \in\left(l_{1}(\widehat{F}(r, s), \mathcal{F}, p, u), l_{1}\right)$ if and only if 4.2, 4.10, 4.11 hold.

Theorem 4.2. Let $1<p_{k} \leq H<\infty$, for all $k \in \mathbb{N}$. Then we have

i) $A=\left(a_{n k}\right) \in\left(l(\widehat{F}(r, s), \mathcal{F}, p, u), l_{\infty}\right)$ if and only if 4.1, 4.2, 4.4 hold.

ii) $A=\left(a_{n k}\right) \in(l(\widehat{F}(r, s), \mathcal{F}, p, u), c)$ if and only if 4.1, 4.2, 4.4, 4.6 hold.

iii) $A=\left(a_{n k}\right) \in\left(l(\widehat{F}(r, s), \mathcal{F}, p, u), c_{0}\right)$ if and only if 4.1, 4.2, 4.4, 4.6 with $\widetilde{a}_{k}=0$ hold

iv) $A=\left(a_{n k}\right) \in\left(l(\widehat{F}(r, s), \mathcal{F}, p, u), l_{1}\right)$ if and only if 4.1, 4.2, 4.5 hold.

Theorem 4.3. i) $A=\left(a_{n k}\right) \in\left(l_{\infty}(\widehat{F}(r, s), \mathcal{F}, p, u), l_{\infty}\right)$ if and only if 4.2, 4.3, 4.4 with $q=1$ hold.

ii) $A=\left(a_{n k}\right) \in\left(l_{\infty}(\widehat{F}(r, s), \mathcal{F}, p, u), c\right)$ if and only if 4.2,4.3, 4.6, 4.7 hold.

iii) $A=\left(a_{n k}\right) \in\left(l_{\infty}(\widehat{F}(r, s), \mathcal{F}, p, u), c_{0}\right)$ if and only if 4.2, 4.3, 4.8 hold.

iv) $A=\left(a_{n k}\right) \in\left(l_{\infty}(\widehat{F}(r, s), \mathcal{F}, p, u), l_{1}\right)$ if and only if 4.2, 4.3, 4.12 hold. 


\section{SOME GEOMETRIC PROPERTİES OF $l(\widehat{F}(r, s), \mathcal{F}, p, u)$}

In the present chapter, we investigate some geometric properties of the space $l(\widehat{F}(r, s), \mathcal{F}, p, u)$. Firstly, let us define some geometric properties of the spaces. let $(X,\|\|$.$) be a normed space and let S(X)$ and $B(X)$ be the unit sphere and unit ball of $X$, respectively.

A Banach space $X$ is said to have the Banach- Saks property if every bounded sequence $\left(x_{n}\right)$ in $X$ admits a subsequence $\left(z_{n}\right)$ such that the sequence $\left(t_{k}(z)\right)$ is convergent in the norm in $X[12]$, where

$$
t_{k}(z)=\frac{1}{k+1}\left(z_{0}+z_{1}+\ldots+z_{k}\right)
$$

$\forall k \in \mathbb{N}$. A Banach space $X$ is said to have the weak Banach-Saks property whenever given any weakly null sequence $\left(x_{n}\right)$ in $X$ and there exists a subsequence $\left(z_{n}\right)$ of $\left(x_{n}\right)$ such that the sequence $\left(t_{k}(z)\right)$ is strongly convergent to zero.

Remark 5.1. In [18] Garcia-Falet introduced the following coefficient,

$$
R(X)=\sup \left\{\liminf _{n \rightarrow \infty}\left\|x_{n}+x\right\|:\left(x_{n}\right) \subset B(X), x_{n} \stackrel{w}{\rightarrow} 0\right\}
$$

Remark 5.2. A Banach space $X$ with $R(X)<2$ has a weak fixed paint property [19]. Let $1<p<\infty$. A Banach space $X$ is said to have the Banach-Saks type $\mathrm{p}$ or the property $(B S)_{p}$ if every null sequence $\left(x_{k}\right)$ has a subsequence $\left(x_{k_{l}}\right)$ such that for some $c>0$

$$
\left\|\sum_{l=0}^{n} x_{k_{l}}\right\|<c(n+1)^{\frac{1}{p}}
$$

for all $n \in \mathbb{N}$.[20]

Now, we may give the following results related to some geometric properties of the space $l(\widehat{F}(r, s), \mathcal{F}, p, u)$, where $1<p_{k} \leq H<\infty$, for all $k \in \mathbb{N}$.

Theorem 5.1. Let $1<p_{k} \leq H<\infty$ for all $k \in \mathbb{N}$. Then the spacel $(\widehat{F}(r, s), \mathcal{F}, p, u)$ has the Banach-Saks type $p$.

Proof. Let $\left(\varepsilon_{n}\right)$ be a sequence of positive numbers for which $\sum \varepsilon_{n} \leq \frac{1}{2}$, and let $\left(x_{n}\right)$ be a weakly null sequence in $B(l(\widehat{F}(r, s), \mathcal{F}, p, u))$. Set $a_{0}=x_{0}=0$ and $a_{1}=x_{n_{1}}=x_{1}$. 
Then there exists $m_{1} \in \mathbb{N}$ such that

$$
\left\|\sum_{i=m_{1}+1}^{\infty} a_{1}(i) e^{i}\right\|_{l(\widehat{F}(r, s), \mathcal{F}, p, u)}<\varepsilon_{1}
$$

since $\left(x_{n}\right)$ being a weakly null sequence implies $x_{n} \rightarrow 0$ coordinatewise, there is an $n_{2} \in \mathbb{N}$ such that

$$
\left\|\sum_{i=0}^{m_{1}} x_{n}(i) e^{i}\right\|_{l(\widehat{F}(r, s), \mathcal{F}, p, u)}<\varepsilon_{1},
$$

when $n \geq n_{2}$. Set $a_{2}=x_{n_{2}}$. Then there exists an $m_{2}>m_{1}$ such that

$$
\left\|\sum_{i=m_{2}+1}^{\infty} a_{2}(i) e^{i}\right\|_{l(\widehat{F}(r, s), \mathcal{F}, p, u)}<\varepsilon_{2} .
$$

Again using the fact that $x_{n} \rightarrow 0$ coordinatewise, there exists an $n_{3} \geq n_{2}$ such that

$$
\left\|\sum_{i=0}^{m_{2}} x_{n}(i) e^{i}\right\|_{l(\widehat{F}(r, s), \mathcal{F}, p, u)}<\varepsilon_{2},
$$

when $n \geq n_{3}$. If we continue this process, we can find two increasing subsequences $\left(m_{i}\right)$ and $\left(n_{i}\right)$ such that

$$
\left\|\sum_{i=0}^{m_{j}} x_{n}(i) e^{i}\right\|_{l(\widehat{F}(r, s), \mathcal{F}, p, u)}<\varepsilon_{j},
$$

for each $n \geq n_{j+1}$ and

$$
\left\|\sum_{i=m_{j}+1}^{\infty} a_{j}(i) e^{i}\right\|_{l(\widehat{F}(r, s), \mathcal{F}, p, u)}<\varepsilon_{j},
$$

where $b_{j}=x_{n_{j}}$. Hence

$$
\begin{gathered}
\left\|\sum_{j=0}^{n} a_{j}\right\|_{l(\widehat{F}(r, s), \mathcal{F}, p, u)}= \\
\left\|\sum_{j=0}^{n}\left(\sum_{i=0}^{m_{j-1}} a_{j}(i) e^{i}+\sum_{i=m_{j-1}+1}^{m_{j}} a_{j}(i) e^{i}+\sum_{i=m_{j}+1}^{\infty} a_{j}(i) e^{i}\right)\right\|_{l(\widehat{F}(r, s), \mathcal{F}, p, u)}
\end{gathered}
$$




$$
\begin{gathered}
\leq\left\|\sum_{j=0}^{n}\left(\sum_{i=0}^{m_{j-1}} a_{j}(i) e^{i}\right)\right\|_{l(\widehat{F}(r, s), \mathcal{F}, p, u)}+\left\|\sum_{j=0}^{n}\left(\sum_{i=m_{j-1}+1}^{m_{j}} a_{j}(i) e^{i}\right)\right\|_{l(\widehat{F}(r, s), \mathcal{F}, p, u)} \\
+\left\|\sum_{j=0}^{n}\left(\sum_{i=m_{j}+1}^{\infty} a_{j}(i) e^{i}\right)\right\|_{l(\widehat{F}(r, s), \mathcal{F}, p, u)} \\
\leq\left\|\sum_{j=0}^{n}\left(\sum_{i=m_{j-1}+1}^{m_{j}} a_{j}(i) e^{i}\right)\right\|_{l(\widehat{F}(r, s), \mathcal{F}, p, u)}+2 \sum_{j=0}^{n} \varepsilon_{j}
\end{gathered}
$$

On the other hand, it can be seen that $\|x\|_{l(\widehat{F}(r, s), \mathcal{F}, p, u)}<1$. Therefore, we have that

$$
\begin{gathered}
\left\|\sum_{j=0}^{n}\left(\sum_{i=m_{j-1}+1}^{m_{j}} a_{j}(i) e^{i}\right)\right\|_{l(\widehat{F}(r, s), \mathcal{F}, p, u)}^{p_{k}}= \\
=\sum_{j=0}^{n} \sum_{i=m_{j-1}+1}^{m_{j}}\left[u_{k} F_{k}\left(\left|r \frac{f_{i}}{f_{i+1}} a_{j}(i)+s \frac{f_{i+1}}{f_{i}} a_{j}(i-1)\right|\right)\right]^{p_{k}} \\
\leq \sum_{j=0}^{n} \sum_{i=0}^{\infty}\left[u_{k} F_{k}\left(\left|r \frac{f_{i}}{f_{i+1}} a_{j}(i)+s \frac{f_{i+1}}{f_{i}} a_{j}(i-1)\right|\right)\right]^{p_{k}} \leq(n+1) .
\end{gathered}
$$

Hence, we obtain

$$
\left\|\sum_{j=0}^{n}\left(\sum_{i=m_{j-1}+1}^{m_{j}} a_{j}(i) e^{i}\right)\right\|_{l(\widehat{F}(r, s), \mathcal{F}, p, u)} \leq(n+1)^{p_{k}} .
$$

By using the fact that

$$
1 \leq(n+1)^{\frac{1}{p_{k}}}
$$

for all $n \in \mathbb{N}$ and $1<p_{k}<\infty$, we have

$$
\left\|\sum_{j=0}^{n} a_{j}(i)\right\|_{l(\widehat{F}(r, s), \mathcal{F}, p, u)} \leq(n+1)^{\frac{1}{p_{k}}}+1 \leq 2(n+1)^{\frac{1}{p_{k}}} .
$$

Hence, the space $l(\widehat{F}(r, s), \mathcal{F}, p, u)$ has the Banach-Saks type $p$. 
Remark 5.3. Note that $R(l(\widehat{F}(r, s), \mathcal{F}, p, u))=R\left(l_{p}\right)=2^{\frac{1}{p}}$, since $l(\widehat{F}(r, s), \mathcal{F}, p, u)$ is linearly isomorphic to $l_{p}$. Thus, by Remarks 5.2 and 5.3 we have the following theorem.

Theorem 5.2. The space $l(\widehat{F}(r, s), \mathcal{F}, p, u)$ has the weak fixed point property, where $1<p_{k} \leq H<\infty$, for all $k \in \mathbb{N}$.

\section{R E F E R E N C E S}

1. Z.U. Ahmad and M. Mursaleen: Köthe-Toeplitz duals of some new sequence spaces and their matrix maps. Publ. Inst. Math. (Belgr.) 42 (1987),57-61.

2. B. Altay and F. BAŞAR: The matrix domain and the fine spectrum of the difference operator $\Delta$ on the sequence space $\ell_{p},(0<p<1)$. Commun. Math. Anal. 2:2 (2007), 1-11.

3. F. BAŞAR: Summability Theory and Its Applications, Bentham Science Publishers. e-books, Monographs, xi+405 pp., İstanbul, (2012), ISB:978-1-60805-252-3.

4. F. BAŞAR and B. Altay: On the space of sequences of p-bounded variation and related matrix mappings. Ukrainian Math. J. 55:1 (2003), 136-147.

5. M. BAŞARIR, F. BAŞAR and E. E. KARA: On the Fibonacci Difference Null and Convergent Sequences. arXiv:1309.0150

6. M. CANDAN: A new aproach on the spaces of generalized Fibonacci difference null and convergent sequences. Math. Aeterna 1:5 (2015),191-210.

7. M. CANDAN and K. Kayaduman: Almost convergent sequence space derived by generalized Fibonacci matrix and Fibonacci core. Brithish J. Math. Comput. Sci. 7 (2015), 150-167.

8. M. CANDAN and E. E. KARA: A study on topological and geometrical characteristics of new Banach sequence spaces. under communication.

9. M. CANDAN and G. KILINÇ: A different look for paranormed Riesz sequence space derived by Fibonacci Matrix. Konuralp Journal of Mathematics 3:2 (2015), 62-76.

10. B. Choudhary and S. NANDA: Functional Analysis with applications. John Wiley and Sons, New Delhi, İndia, 1989.

11. R. Çolak, M. Et and E. Malkowsky : Some Topics of Sequence Spaces. Lecture Notes in Mathematics, Firat Univ. Press (2004) 1-63 ISBN: 975-3940386-6.

12. J. Diestel: Sequences and Series in Banach Spaces. Springer, New York, NY, USA, 1984.

13. J. GARCIA-FALSET: Stability and fixed points for nonexpansive mappings. Houst. J. Math. 20 (1994), 495-506.

14. E. E. KARA: Some topological and geometrical properties of new Banach sequence spaces.J. Inequal. Appl. 38 (2013), 1-15.

15. E. E. KARA, M. BAŞARIR and M. Mursaleen: Compact operators on the Fibonacci difference sequence spaces $l_{p}(\widehat{F})$ and $l_{\infty}(\widehat{F})$. 1st International Eurasian Conf. on Math. Sci. and Appl. Prishtine-Kosovo, (2012), September 3-7. 
16. M. KIRIŞÇI and F. BAŞAR: Some new sequence spaces derived by the domain of generalized difference matrix. Comput. Math. Appl. 60:5 (2010), 1299-1309.

17. H. Kizmaz: On certain sequence spaces. Canad. Math. Bull. 24:2 (1981), 169176.

18. J. GARCIA-FAlSET: Stability and fixed points for nonexpansive mappings. Houst. J. Math. 20 (1994), 495-506.

19. J. GARCIA- FALSET: The fixed point property in Banach Spaces with the NUSproperty. J. Math. Anal. Appl. 215 (1997), 532-542.

20. H. Knaust: Orlicz sequence Spaces of Banach-Saks Type. Arch. Math. 59 (1992), $562-565$.

21. I. J. Maddox: Paranormed sequence spaces generated by infinite matrices. Proc. Cambridge Philos. Soc. 64 (1968), 335-340.

22. I. J. MADdox: Spaces of strongly summable sequences. Quart. J. Math. 18:2 (1967), 345-355.

23. I. J. MAdDox: Continuous and Köthe-Toeplitz duals of certain sequence spaces. Proc. Camb. Philos. Soc. 65 (1965), 431-435.

24. E. Malkowsky: Absolute and ordinary Köthe-Toeplitz duals of some sets of sequences and matrix transformations. Publ. Inst. Math. (Belgr.) 46 (1989), 97103.

25. H. NAKAnO: Modulared sequence spaces. Proc. Japan Acad. 27:2 (1951), 508-512.

26. K. RAJ, S. PANDOH and S. JAMWAL: Fibonacci difference sequence spaces for modulus functions. Le Matematiche,LXX 1 (2015),137-156.

27. S. Simons: The sequence spaces $\ell\left(p_{v}\right)$ and $m\left(p_{v}\right)$. Proc. London Math. Soc. 15:3 (1965), 422-436.

28. M. Stieglitz and H. Tietz: Matrix transformationen von folgenräumen eine ergebnisübersicht. Math. Z. 154 (1977), 1-16.

29. A. Wilansky: Summability through functional analysis. North- Holland Math. Stud., 1984.

Gülsen Kılınç

Faculty of Education

Department of Elementary Education

Adıyaman University,

The University Campus

02040-Adiyaman/TURKEY

gkilinc@adiyaman.edu.tr

Murat Candan

Department of Mathematics

Faculty of Arts and Sciences,s

İnönü University,

The University Campus,

44280-Malatya/TURKEY

murat. candan@inonu.edu.tr 